Full length article

\title{
STABILIZED NUMERICAL METHODS FOR THE TWO KINDS OF PROBLEMS OF INCOMPRESSIBLE FLUID FLOWS
}

Shahid Hussain', " and Sajid Hussain²

1. School of Mathematical Sciences, East China Normal University China

2. School of Mathematical Sciences, Xian Jiaotong University Xian China

\section{ABSTRACT}

A mixed finite element method (MFEM) stabilized for the two kinds of problems related to the incompressible fluid flow is demonstrated. In the first kind, the Newtonian fluid flow is illustrated with the MFEM and considered discontinuous scheme. Initially, the model equations are considered nonlinear and un-stabilize. The model equations are solved for linear terms with the special technique first and then the model equation with the extra added term is utilized later to stabilize the model equations. A steady-state viscoelastic Oseen fluid flow model with Oldroyd-B type formulations was demonstrated in the second kind of problem with SUPG method. The nonlinear problems are linearized through the Oseen scheme. Numerical results for both the model equations are given and compared. The SUPG method is found more suitable and active.

KEYWORDS: Finite Element Method, Lowest Equal Order Elements, DG method, SUPG technique.

*Corresponding author: (Email: 52150601025@std.ecnu.edu.cn, Phone: 0086-19946036403)

\section{INTRODUCTION}

We are interested to discuss only the nonsteady state fluid flow problems under the 2dimensional bounded and connected domain. In this case, for the incompressible time independent model equations are standard under applied forces and stresses as follows $[1,2]$

$\rho_{\text {total }}=P I+\rho_{N+} \rho$. Where $\lambda$ represents the Wesenberg number which is well known in the given literature and the term $g_{a}(\rho . \nabla M)$ is defined as:

$\lambda g_{a}(\rho . \nabla M)=\frac{1-a}{2}(\rho . \nabla M)+\left((\nabla M)^{T} \rho-\frac{1-a}{2}(\rho . \nabla M) \rho\right)$

We can write the model equation for given forces $f$ with the given parameter related to the materials as: $(M . \nabla) M-\nabla \rho_{\text {total }}=f$. while the Oldroyd model is defined by $\rho_{\text {total }}=P I+\rho_{N+} \rho$. This denotes the total stress

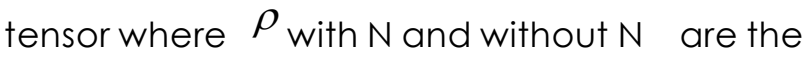
Newtonian and viscoelastic parts respectively. The model equation for these important issues are given below in detail formulation. 


\section{$1.1 \quad$ Model Problem}

The model equations, without time dependent ter$m$ under the open domain $\Omega$ is considered. The Dirichlet boundary condition with homogeneous nature is regarded for the fluid velocity $\mathbf{M}$ in the nonnewtonian form; The model equations are given as:

$$
\begin{gathered}
\rho+\lambda(\mathbf{M} \cdot \nabla) \rho+\lambda g_{a}(\rho, \nabla \mathbf{M})-\beta 2 D(\mathbf{M}) \\
=0 \\
-2(-\beta+1) \nabla \cdot D(\mathbf{M})-\nabla \cdot \rho \\
\nabla p=\mathbf{f} \operatorname{in} \Omega, \\
\nabla \cdot \mathbf{M}=0 \quad \operatorname{in} \Omega, \\
\mathbf{M}=0 \text { on } \Gamma,
\end{gathered}
$$

Let us consider pressure $\mathrm{p}$ is zero at the boundary. The solution of the partial differential equations (7)- 9 require boundary conditions to be specified where appropriate [3]. The velocity components are identified along the boundaries where the assumptions of no-slip boundary conditions and nopenetration conditions are applied so that both velocity components vanish there. The inlet and outlet boundaries requirements are applied smartly for the vector fields [4]. So that for the calculation, no boundary conditions are used for the stresses here. For the literature review, the well-posedness of the model equations or about the study of existence and uniqueness of the equations $(7)-(9)$, we would like to motivate the reader to see [5] for further guidance.

In the analysis part, we are keen to note the linearized form of the given equations. In the numerical solution of the model equations section, we illustrate the conditions

Problem $(\mathrm{O})$ : Solve the problem of $(\rho, \mathbf{M}, p)$ such that

$$
\begin{aligned}
& \rho+\lambda(\mathbf{M} \cdot \nabla) \rho+\lambda g_{a}(\rho, \nabla \mathbf{M}) \\
& -2 \beta D(\mathbf{M})=0 \text { in } \Omega, \\
& \nabla p-2(1-\beta) \nabla \cdot D(\mathbf{M})-\nabla \cdot \rho \\
& =\mathbf{f} \operatorname{in} \Omega \\
& \nabla \cdot \mathbf{M}=0 \mathrm{in} \Omega \text {, } \\
& \mathbf{M}=0 \text { on } \Gamma(12)
\end{aligned}
$$

We understand with the following assumption for the additional term for velocity $\mathbf{M}(x)$ which can be searched in the [6] for any positive constant $M>0$. This positive constant depends only on the smooth domain, and also it is independent of the other constants and grid size parameters,

$$
\begin{gathered}
\mathbf{M} \in H_{0}^{1}(\Omega), \quad \nabla \cdot \mathbf{M}=0, \quad\|\mathbf{M}\|_{\infty} \leq M, \\
\|\nabla \mathbf{M}\|_{\infty} \leq M<\infty .
\end{gathered}
$$

\subsection{Variational formulation}

We are keen to introduce some basic required symbols. These are classical for $m \in N$ the norm is related to the special type of Hilbert space known as Sobolve spaces $W^{n, p}(\Omega)$ by $\|\cdot\|_{W^{n, q}}$, with the given special case $W^{m, 2}(\Omega)$, this can be written as $[7,8]$ :

$$
\begin{aligned}
X= & H_{0}^{1}(\Omega)^{2}=\left\{\mathbf{v} \in H^{1}(\Omega)^{2}, \mathbf{v}=\mathbf{0} \text { Hilbertspce }\right\} \\
Q= & L^{2}(\Omega)=\left\{q \in L^{2}(\Omega), \quad\right\} \\
S_{\rho}= & \left\{\rho=\left(\rho_{i j}\right) ; \rho_{i j}=\rho_{j i}: \rho_{i j} \in L^{2}(\Omega) ; i, j=1,2\right\} \\
& \cap\left\{\rho=\left(\rho_{i j}\right) ; \mathbf{M} \cdot \nabla \rho \in L^{2}(\Omega)^{2 \times 2}, X\right\}
\end{aligned}
$$

In order to find the appropriate weak formulation of the given Problem $(O)$. To find unknowns as:

$$
\begin{aligned}
(\rho, \sigma)+\lambda((\mathbf{M} \cdot \nabla) \rho, \sigma) & \\
+\lambda\left(g_{a}(\rho, \nabla \mathbf{M}), \sigma\right)-\beta((\sigma, \mathbf{M})) & =0 \\
\forall \sigma \in S_{\rho}, & (14) \\
-(\nabla \cdot \mathbf{v}, p)+(1-\beta)(T(\mathbf{M}), T(\mathbf{v}))+(\rho, D(\mathbf{v})) & (15) \\
& =(\mathbf{v}, \mathbf{f}) \forall \mathbf{v} \in X \\
(\nabla \cdot \mathbf{M}, q) & =0
\end{aligned}
$$

The solution generated is not a minimum in the given spaces, but is indeed a saddle point. The standard existence of this saddle point is satisfied by the chosen finite elements or Lagrangian polynomials for the velocity and pressure in discrete form, if it can be proved that a compatibility condition for velocity $\mathbf{v}$ and pressure $\mathrm{p}$ is satisfied. This important condition (inf-sup or LBB) states that $[7,9]$

$$
\sup _{\mathbf{v} \in X} \frac{(q, \nabla \cdot \mathbf{v})}{\|\mathbf{v}\|_{1}} \geq C\|q\| \forall q \in Q,
$$

It requires the roundedness of the variational operator and restricts the choices for the approximating spaces.

For further detail formulation, The multiplication of $\beta$ with the equation $\sqrt{16}$ and add together with (14) deals with the bilinear form $\mathcal{A}$ and $B$ :

$$
\begin{aligned}
& \mathcal{A}((\rho, \mathbf{M}, p),(\sigma, \mathbf{v}, q)) \\
& =(\rho, \sigma)+\lambda\left(g_{a}(\rho, \nabla \mathbf{M}), \sigma\right)-\beta(D(\mathbf{M}), \sigma) \\
& +\beta(\rho, D(\mathbf{v}))+4 \alpha(1-\alpha)(D(\mathbf{M}), D(\mathbf{v})) \\
& -\alpha(\nabla \cdot \mathbf{v}, p)+\beta(q, \nabla \cdot \mathbf{M}) \\
& \lambda B(\mathbf{M}, \rho, \sigma)=\lambda((\mathbf{M} \cdot \nabla) \rho, \sigma) .
\end{aligned}
$$

By using the bilinear form $\mathcal{A}((\cdot, \cdot, \cdot),(\cdot, \cdot, \cdot))$ and $B(\cdot, \cdot, \cdot)$, the equations (14)

$$
\begin{aligned}
& \mathcal{A}((\rho, \mathbf{M}, p),(\sigma, \mathbf{v}, q))+\lambda B(\mathbf{M}, \rho, \sigma) \\
& =\beta(\mathbf{f}, \mathbf{v}) .
\end{aligned}
$$

An equivalent formulation of $(19)$ is given as

$$
\begin{aligned}
& \widetilde{\mathscr{N}}((\rho, \mathbf{M}, p),(\sigma, \mathbf{v}, q))= \\
& 2 \beta(\mathbf{f}, \mathbf{v}), \quad \forall(\sigma, \mathbf{v}, q) \in S_{\rho} \times X \times Q
\end{aligned}
$$


where

$$
\begin{aligned}
& \widetilde{\mathscr{N}}((\rho, \mathbf{M}, p),(\mathbf{v}, \sigma,, q)) \\
& =\mathcal{A}((\rho, \mathbf{M}, p),(\sigma, \mathbf{v}, q))+\lambda B(\mathbf{M}, \rho, \sigma) .
\end{aligned}
$$

\section{Discontinuous FE}

The $D G$ method and $S U P G$ method are mostly utilized in viscoelastic fluid flow problems to find the approximate solutions of discontinuous stress strain, In this work, the $D G$ and SUPG methods are used and compared. Let us consider the elements of a triangulation in domain $\Omega$ is denoted as $T^{h}$ i.e., $\widetilde{\Omega}=\left\{\cup K: K \in T^{h}\right\}$. Assume that $r_{\min }$ and $r_{\max }$ are the maximum and minimum diameter of the elements of $T^{h}$, respectively. This will allow us to simplify notation, and use mesh size $h$ to represent the characteristic length of all the triangular elements of $T^{h}$ [8]. Then there exist positive constants, i.e.,

$$
r_{\min } h \leq h_{K} \leq r_{\max } \rho_{K}
$$

Accordingly, we define discrete subspaces for the FE approximation of the equation (20)

$$
\begin{aligned}
X^{h} & :=\left\{\mathbf{v} \in X \cap C^{0}(\widetilde{\Omega})^{2} ; \forall K \in T^{h}\right\}, \\
Q^{h} & :=\left\{q \in Q \cap C^{0}(\widetilde{\Omega}) ; q_{\mid K} \in P_{1}(K) ;\right\}, \\
S_{\rho}^{h} & :=\left\{\sigma \in S_{\rho} ; \sigma_{\mid K} \in P_{1}(K)^{2 \times 2} ;\right\},
\end{aligned}
$$

We define $\partial K^{-}(\mathbf{M})=\{x \in \partial K ; \mathbf{M}(x) \cdot \mathbf{n}(x)<0\}$ where $\partial K$ is the boundary of $K \in T^{h}$ and $\mathbf{n}$, and

$$
\begin{aligned}
\Gamma^{h} & =\left\{\cup \partial K: K \in T^{h}\right\} \backslash \Gamma, \\
\rho^{ \pm}(\mathbf{M}(x)) & =\lim _{\varepsilon \rightarrow 0} \rho(x \pm \varepsilon \mathbf{M}(x)) .
\end{aligned}
$$

Also, for any $(\rho, \sigma) \in \prod_{K \in T^{h}}\left[H^{1}(K)\right]^{4}$, we define

$$
\begin{aligned}
(\rho, \sigma) & =\sum_{K^{2} \in T^{h}}(\rho, \sigma)_{K}, \\
\left\langle\rho^{ \pm}, \sigma^{ \pm}\right\rangle & =\sum_{K \in T^{h}} \int_{\partial K^{-}(\mathbf{M})}\left(\rho^{ \pm}(\mathbf{M}), \sigma^{ \pm}(\mathbf{M})\right) d s, \\
\left\langle\left\langle\rho^{ \pm}\right\rangle\right\rangle_{h, \mathbf{M}}^{2} & =\left\langle\rho^{ \pm}, \rho^{ \pm}\right\rangle_{h, \mathbf{M}}, \\
\|\rho\|_{0, \Gamma^{h}} & =\left(\sum_{K \in T^{h}}|\rho|_{0, \partial K}^{2}\right)^{1 / 2} .
\end{aligned}
$$

The term $((\mathbf{M} \cdot \nabla) \rho, \sigma)$ is solved with an operator
$B^{h}$ on $\left(X^{h}, S_{\rho}^{h}, S_{\rho}^{h}\right)$, which is stated in [9] by

$$
\begin{aligned}
B^{h}\left(\mathbf{M}, \rho^{h}, \sigma^{h}\right)= & \left.\left((\mathbf{M} \cdot \nabla) \rho^{h}, \sigma^{h}\right)_{h} \mathbf{M} \rho^{h}, \sigma^{h}\right) \\
& +(1 / 2)\left(\nabla \cdot+\left\langle\rho^{h+}\right.\right. \\
& \left.-\rho^{h-}, \sigma^{h+}\right\rangle_{h, \mathbf{M}}, \\
= & -\left((\mathbf{M} \cdot \nabla) \sigma^{h}, \rho^{h}\right)_{h} \\
& -(1 / 2)\left(\nabla \cdot \mathbf{M} \sigma^{h}, \rho^{h}\right)+ \\
& \left\langle\rho^{h-}, \sigma^{h-}-\sigma^{h+}\right\rangle_{h, \mathbf{M}}, \\
= & \left((\mathbf{M} \cdot \nabla) \rho^{h}, \sigma^{h}\right)_{h} \\
& +\left\langle\rho^{h+}-\rho^{h-}, \sigma^{h+}\right\rangle_{h, \mathbf{M}}, \\
& \text { if } \nabla \cdot \mathbf{M}=0 .
\end{aligned}
$$

Thus,

$$
\begin{aligned}
& B^{h}\left(\mathbf{M}, \rho^{h}, \rho^{h}\right)= \\
& (1 / 2)\left\langle\left\langle\rho^{h+}-\rho^{h-}\right\rangle\right\rangle_{h, \mathbf{M}}^{2} \geq 0 .
\end{aligned}
$$

The main objective of this work is to find the solution of viscoelastic fluid flow problems with a mixed FE method by applying the FE triples as a polynomial space in discrete formulation due to the deficiency of the inf-sup condition the scheme is not stable more. Thus, it is important to add a stabilization term to circumvent the inf-sup condition . This idea was proposed and well-defined for the Stokes problem in the finite element method $[10,11]$. We are interested in working with the same idea to find the approximate solution to the viscoelastic fluid flow problems. We think that this idea is new and very useful for viscoelastic fluid flow problems. Specifically, this technique is new for the stable solution of the viscoelastic fluid flow problems, and in the existing research, there is no such technique available. Indeed this stabilization method is not expensive as the existing stabilization methods. For more work, to ensure the well-posedness of the confirming finite element methods, we introduce asymmetric, non-trivial, and penalty terms which add the penalizing parameter. For further investigations and brief study see the references we have cited $[12,13]$

$$
H\left(p^{h}, q^{h}\right)=\left((I-\Pi) p^{h},(I-\Pi) q^{h}\right),
$$

where $\Pi: L^{2}(\Omega) \rightarrow \mathbb{R}_{0}$ is the standard piecewise constant space $\mathbb{R}_{0}$. The reversed operator $\Pi$ has the following feature

$$
\begin{gathered}
\|\Pi p\|_{0} \leq k\|p\|_{0}, \\
\|p-\Pi p\| \leq k h\|p\| .
\end{gathered}
$$

In this work, $k$ means as a positive constant, which is free of any mesh size value. 


\subsection{Analytical solution test}

The verifications with convergence rates are demonstrated by considering fluid flow across a square domain with a known solution. To verify the numerical values of the new formulated scheme, some one can considered the standard FE triples for the unknowns $[14,15]$. Many researchers used this experimental pattern for the Stokes and the Navier-Stokes equation.

In the given example, the known function $\mathbf{m}(x)$ is chosen to be the true values of the velocity $\mathbf{M}$ $[16,17]$. However, the true values of the model equations for velocity $\mathbf{M}=\left(u_{1}, u_{2}\right)$, pressure $p$ and polymeric stress $\rho$ is given by

$$
\left\{\begin{aligned}
\mathbf{M} & =\left(\begin{array}{r}
-10\left(x^{4}-2 x^{3}+x^{2}\right)\left(2 y^{3}-3 y^{2}+y\right) \\
10\left(2 x^{3}-3 x^{2}+x\right)\left(y^{4}-2 y^{3}+y^{2}\right) \\
p
\end{array}=-10.0(2 x-1)(2 y-1)\right. \\
\rho & =2 \beta D(\mathbf{M})
\end{aligned}\right.
$$

The right-hand sides, initial and boundary conditions are derived by model $\operatorname{Problem}(O)$ with the parameters value $a=0, \lambda=5.0$ and

We illustrated the specific features of the lowest equal order MFE method for the Oseen nonnewtonian. We included the $H^{1}$-norm for fluid velocity, $L^{2}$-norm error for fluid pressure, and $L^{2}$-norm error for stress, respectively, with the variable spacing such as $h=1 / 4,1 / 8,1 / 16,1 / 64$. In the computations of the errors for the standard MFE with $P_{1 b}-P_{1}-P_{d c}^{1}$ pairs. The table gives the DG method. let's say : $\left\|u-u^{h}\right\|_{0}=A,\left\|u-u^{h}\right\|_{1}=B$, $\left\|p-p^{h}\right\|_{0}=C$ and $\left\|\tau-\tau^{h}\right\|_{0}=D$

\begin{tabular}{|c|c|c|c|c|}
\hline$h$ & $A$ & $B$ & $C$ & $D$ \\
\hline $1 / 4$ & 0.017 & 0.226 & 0.22 & 0.109 \\
\hline $1 / 8$ & 0.005 & 0.102 & 0.062 & 0.033 \\
\hline $1 / 16$ & 0.001 & 0.048 & 0.019 & 0.010 \\
\hline
\end{tabular}

Table given to demonstrate the SUPG method

\begin{tabular}{|c|c|c|c|c|}
\hline$h$ & $A$ & $B$ & $C$ & $D$ \\
\hline $1 / 4$ & 0.018 & 0.236 & 0.329 & 0.117 \\
\hline $1 / 8$ & 0.004 & 0.110 & 0.062 & 0.043 \\
\hline $1 / 16$ & 0.001 & 0.038 & 0.019 & 0.010 \\
\hline
\end{tabular}

\section{Conclusions}

We have given the two methods for the stabilization techniques by using finite element lowest equal polynomial elements. Since the model equations were non linear Partial Differential equations. We first made these model equations linear with the help of Oseen technique and formulated approximate numerical solutions. Two different numerical methods were demonstrated by considering the same finite elements for the same stabilized formulation. From the numerical studies, the results of the SUPG are more accurate than the DG method. For results and geometrical configuration, readers can see the references given at the end of this manuscript.

\section{Conflicts of interest}

The authors declare no any conflict of interest.

\section{Data availability}

Not applicable.

\section{Code availability \\ Available.}

\section{Authors contributions}

Shahid hussain: Conceptualization, methodology, analysis and writing original draft.

Sajid Hussain: Methodology editing and reviewing.

\section{References}

[1] J. Baranger, S. Wardi, Numerical analysis of a FEM for a transient viscoelastic flow, Comput. Methods Appl. Mech. Engrg., 125, (1995) 171-185.

[2] K. Najib, D. Sandri, On a decoupled algorithm for solving a finite element problem for the approximation of viscoelastic fluid flow, Numer. Math., 72, (1995) 223-238.

[3] D. Sandri, Finite element approximation of viscoelastic fluid flow: Existence of approximate solutions and error bounds. Continuous approximation of the constraints, SIAM J. Numer. Anal., 31, (1994) 362-377.

[4] E. Fernández-Cara, F. Guillén, R. R. Ortega, Mathematical modeling and analysis of viscoelastic fluids of the Oldroyd Kind, in: Handbook of Numerical Analysis, North-Holland, Amsterdam, (2002) 543-661.

[5] C. Guillopé, J. C. Saut, Existence results for the flow of viscoelastic fluids with a differential constitutive law, Nonlinear Anal., 15, (1990) 849869. 
[6] H. Lee, A multigrid method for viscoelastic fluid flow, SIAM J. Numer. Anal., 42, (2004) 109129 .

[7] V. J. Ervin, H. Lee, Defect correction method for viscoelastic fluid flows at high Weissenberg number, Numer. Meth. PDEs., 22, (2006) 145164.

[8] H. B. Zheng, J. P. Yu, L. Shan, Unconditional error estimates for time dependent viscoelastic fluid flow, Appl. Numer. Math., 119, (2017) 117.

[9] Y. N. He, C. Xie, H. B. Zheng, A Posteriori Error Estimate for Stabilized Low-Order Mixed FEM for the Stokes Equations, Adv. Appl. Math. Mech., (2010) 798-809.

[10] P. B. Bochev, C. R. Dohrmann, M. Gunzburger, Stabilization of low order mixed finite elements for the Stokes equations, SIAM J. Number. Anal., 44, (2006) 82-101.

[11] Y. Li, J. He, A stabilized finite element method based on local polynomial pressure projection for the stationary Navier-Stokes equations, Appl. Numer. Math., 58, (2008) 1503-1514.
[12] H. Jia, K. Li, S. Liu, Characteristic stabilized finite element method for the transient Navier-Stokes equations., Comput. Methods Appl. Mech. Engrg., 199, (2010) 2996-3004.

[13] Z. Li, S. Chen, S. Qu, M. Li, Stabilization of low-order mixed finite elements for the plane elasticity equations, Comput. Math. Appl., 73, (2017) 363-373.

[14] R. A. Adams, Sobolev Space. Pure and Applied Mathematics, vol. 65. Academic Press, New York (1975).

[15] J. M. Marchal, M. J. Crochet, A new mixed finite element for calculating viscoelastic flow, J. Non-Newtonian Fluid Mech., 26, (1987) 77-114.

[16] V. J. Ervin, H. Lee, L. N. Ntasin, Analysis of the Oseen-viscoelastic fluid flow problem, J. Non-Newtonin fluid Mech., 127, (2005) 157168.

[17] C. Guillopé, J. C. Saut, Existence results for the flow of viscoelastic fluids with a differential constitutive law, Nonlinear Anal. TMA., 15, (1990) 849-869. 